\title{
Advection and its influence on the characteristics of sensible and latent heat flux of internal boundary layer
}

\author{
Seiji HAYAKAWA*, Hiroshi TANI*, Haruhiko YAMAMOTO *and Tamon TSUJI**
}

(*Faculty of Agriculture, Yamaguchi University, Yamaguchi, 753, Japan)

(**United department of Agricultural Science,Graduate School of Tottori University, Tottori,680, Japan)

\begin{abstract}
Horizontal inhomogeneity is a common occurrence in the lower layers of the atmosphere. For example,in a land-lake interface problem, and in flows over a finite area of irrigated land, abrupt changes of surface conditions, i.e., roughness, temperature and humidity occur simultaneously, and the profiles are no longer in equilibrium. The principal purpose of this paper is to present these phenomena quantitatively from theory.

The profiles of the diffusion coefficient $(\mathrm{K})$ obtained by use of a gradient Richardson number show its peaks at reasonable levels, and the values of $\mathrm{K}$ and the peak height increase with the scale of a localized heat source(LHS). These characteristics agree qualitatively with observations in the planetary boundary layer.

When air moves from a cool area to a hot one, the horizontal temperature gradient $\partial \mathrm{T} / \partial \mathrm{x}$ becomes positive above the hot area, and the gradient of vertical sensible heat flux $\partial \mathrm{H} / \partial \mathrm{z}$ becomes positive. On the other hand, when air moves from a hot area to a cool one, $\partial \mathrm{T} / \partial \mathrm{x}$ and $\partial \mathrm{H} / \partial \mathrm{z}$ become negative above the cool area.

Key words: advection, boundary layer, diffusion coefficient, flux, stability
\end{abstract}

\section{Introduction}

The surface of the earth is naturally heterogeneous with respect to its roughness, temperature, moisture and other properties. When air moves over such a surface, it is modified continuously by the horizontally varying properties. The degree of this modification depends on the amplitude and areal extent of the surface inhomogeneities as well as the prevailing large scale flow pattern.

The nonlinear two-dimensional steady-state equations for the planetary boundary layer are constructed and solved using Estoque and Bhumralkar's model (1970), which is based on the numerical solution of the steady-state nonlinear meteorological equations for three dimensional flow. In accordance with the similarity theory, thermal stability was assessed in terms of Richardson number(Ri). The evaluation of $\mathrm{Ri}$ is open to question because it is based on the doubtful assumption that the stability condition of the planetary boundary layer is uniform with height. In order to take vertically varying stability conditions into consideration, Hayakawa et al.(1979) suggested using a gradient Richardson number $\left(\mathrm{Ri}_{\mathrm{g}}\right)$ for the stability parameter. In this paper are presented the characteristics of vertical flux profiles of a heterogeneous region obtained by numerical simulation and the results of a comparison of simulated and observed conditions.

\section{Calculation Method}

We consider the problem involving air flow when properties vary along only one direction. Let this direction be along the $\mathrm{x}$ axis. The surface properties which will be allowed to vary are the roughness parameter, the temperature and the mixing ratio. Therefore, the appropriate governing equations are the following: 


$$
\begin{aligned}
& \begin{array}{c}
u \partial u / \partial x+w \partial u / \partial z-f v \\
=-(1 / \rho) \partial P^{\prime} / \partial x+\partial(K \partial u / \partial z) / \partial z \\
u \partial v / \partial x+w \partial v / \partial z+f u \\
=-(1 / \rho) \partial P^{\prime} / \partial y+\partial(K \partial v / \partial z) / \partial z \\
u \partial 0 / \partial x+w \partial 0 / \partial z=\partial(K \partial 0 / \partial z) / \partial z
\end{array}
\end{aligned}
$$

$$
\begin{aligned}
& u \partial q / \partial x+w \partial q / \partial z=\partial(K \partial q / \partial z) / \partial z \\
& \partial u / \partial x+\partial u / \partial z=0 \\
& \partial \pi / \partial z=-g / 0 \\
& \quad \pi=C_{p}\left(P / P_{0}\right)^{k} ; \kappa=R_{c} / C_{p}
\end{aligned}
$$

In these equations, $R_{e}$ is the gas constant for air, $C_{p}$ is the specific heat at constant pressure, $\mathrm{g}$ is gravity and $\mathrm{P}_{0}$ is $1000 \mathrm{hPa}$. The horizontal pressure gradient along $\mathrm{y}, \partial \mathrm{P} / \partial \mathrm{y}$ is to be specified as a known quantity which may vary with altitude. By prescribing this quantity properly, one is able to specify any desired prevailing wind. The $\mathrm{x}$ component, $\partial \mathrm{P} / \partial \mathrm{x}$ is an unknown quantity. The eddy diffusivity can be specified as a function of height in any arbitrary manner. In our calculation, we assumed a particular expression similar to one which was used previously by Blackadar(1962).

\section{Result and Discussion}

One of the most important phenomena caused by advection is the change of air layer properties when the air passes over an imhomogeneous surface. It is important to know how the internal boundary later will develop into the atmosphere. At this point, what boundary layer will be formed, and how will it affect surrounding environmental conditions? In order to understand these phenomena, Fig. 1 shows calculations of temperature fields about an isolated region in which the surface temperature $10^{\circ} \mathrm{C}$ is higher. It is seen that the air layer is influenced in the horizontal and vertical directions to the leeward, as a result of the heat source.

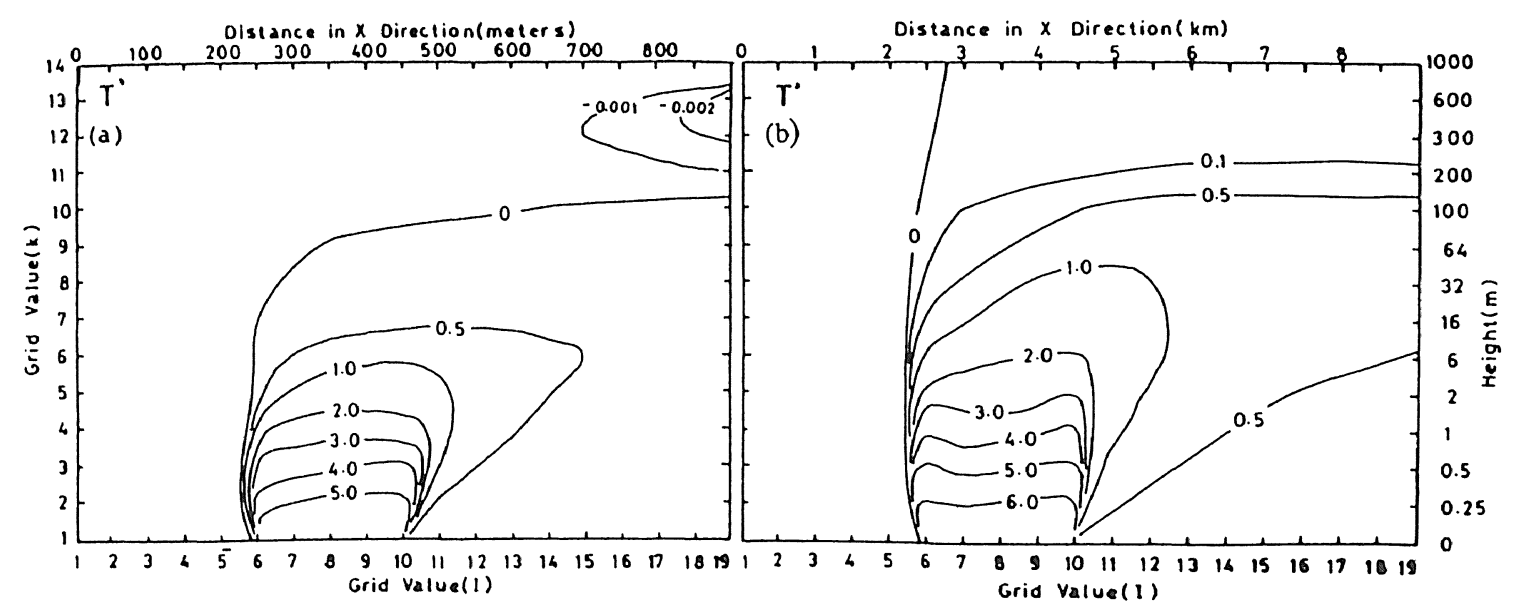

Fig.1 Distributions of perturbation in potential temperature $\theta^{\prime}\left({ }^{\circ} \mathrm{C}\right)$ with $\mathrm{Ri}_{\mathrm{g}}$ for different scales of LHS (a: $L=200 \mathrm{~m} ; b: L=2 \mathrm{~km}, \mathrm{~L}$ : the size of a localized heat source)

(1)What distribution will the stable layer show, and how will the diffusion coefficient change?

Fig.2 shows spatial distribution of Richardson number for some conditions as Fig.1 


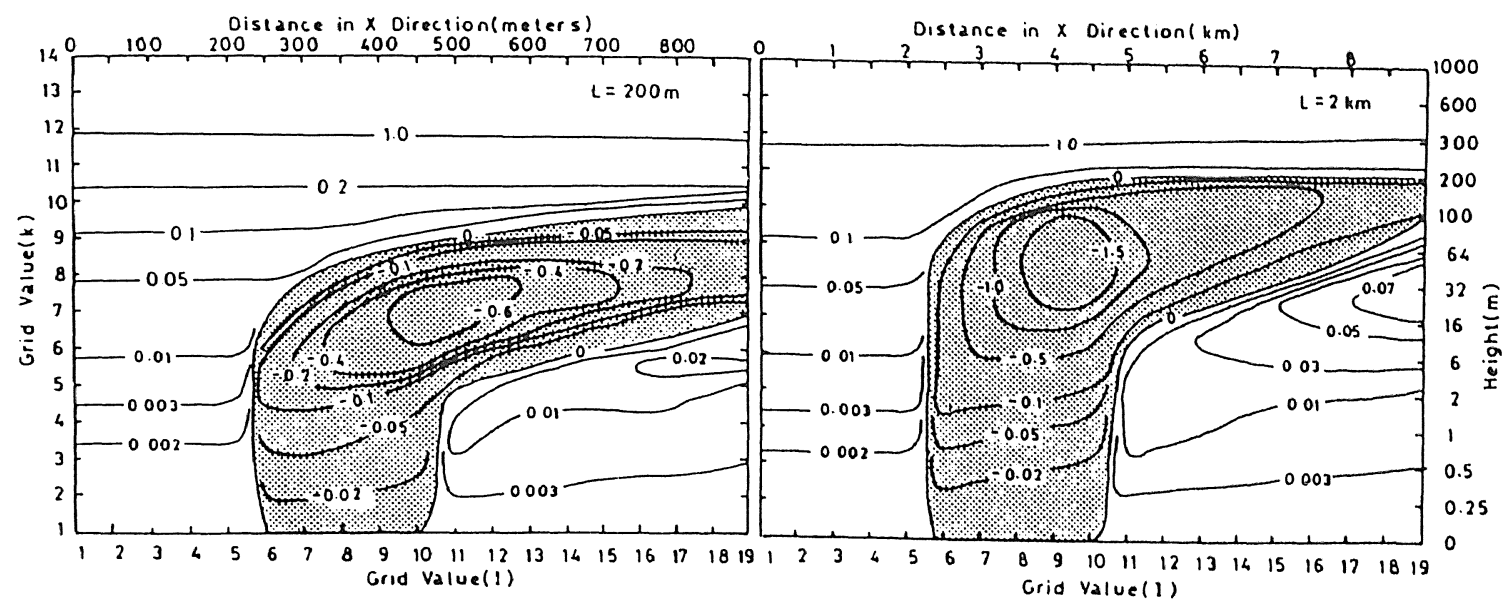

Fig.2 Spatial distributions of Richardson number $\left(\mathrm{Ri}_{\mathrm{g}}\right)$ for $\mathrm{L}=200 \mathrm{~m}$ and $\mathrm{L}=2 \mathrm{~km}$.

The unstable layer is gradually extending far in the atmosphere owing to the hot source effects, and the most unstable layer exists near the height $10 \mathrm{~m} \sim 150 \mathrm{~m}$ leeward of the hot source.

Fig. 3 shows the vertical distribution of the diffusion coefficient with the use of $\mathrm{Ri}_{b}$ and $\mathrm{Ri}_{\mathrm{g}}$. The distribution patterns of diffusion coefficient are simple with the use of $\mathrm{Ri}_{b}$ (bulk Richardson number) such that $\mathrm{K}$ becomes large when unstable and becomes small when stable. The vertical distributions become more complicated with the use of $\mathrm{Ri}_{\mathrm{g}}$. In this case, the stability of each layer is considered and the diffusion coefficient becomes large when the layer becomes unstable. Characteristics of the diffusion coefficient for different layers are affecting each another, and the temperature field is determined by the diffusion coefficient.
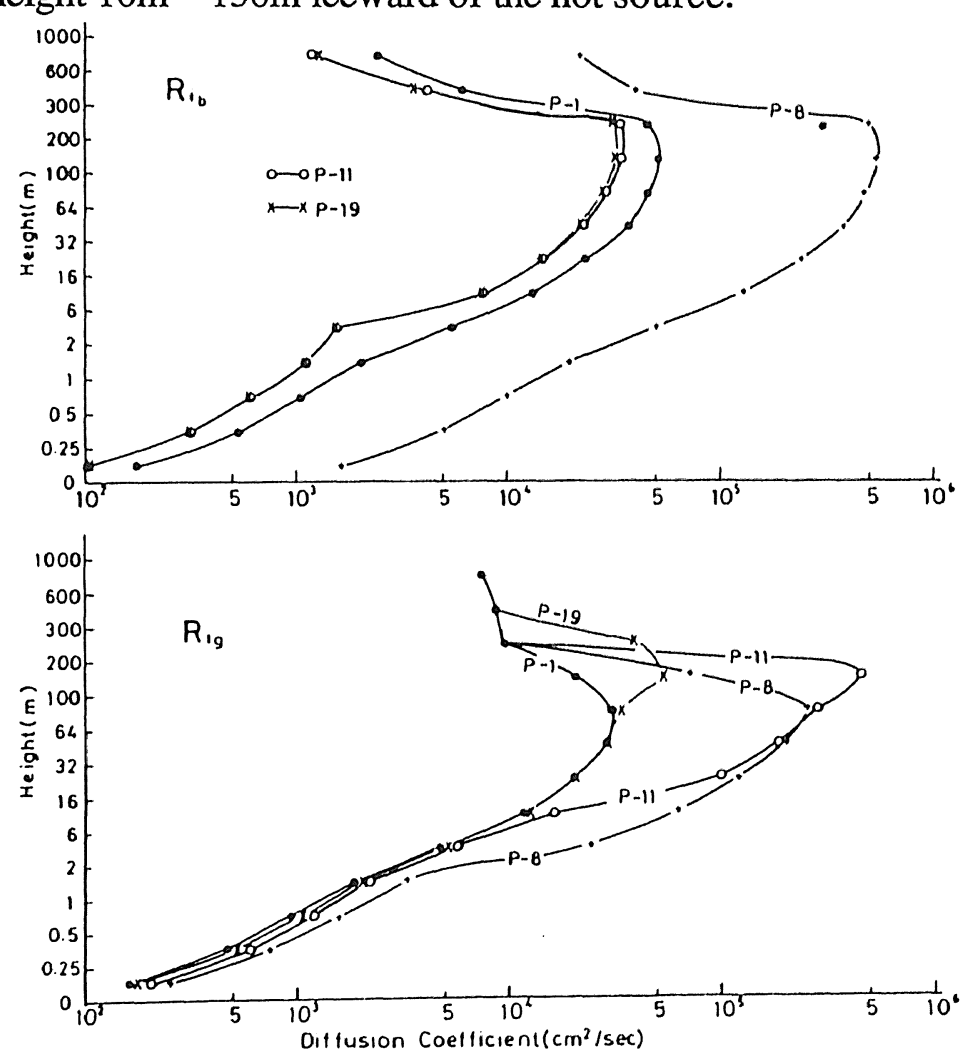

Fig.3 Vertical profiles of the diffusion coefficient over the various grid points along $x$ axis with $\mathrm{L}=2 \mathrm{~km}$. Upper is based on $\mathrm{Ri}_{\mathrm{b}}$ and lower on $\mathrm{Ri}_{\mathrm{g}}$. $\mathrm{P}$ is the horizontal grid point along $\mathrm{x}$ axis.

\section{(2) Characteristics of the flux under advective condition}

The characteristics of vertical profiles of fluxes under advective condition are following from the results of Hayakawa et al. (1978). Once the dry land has formed, evaporation will decrease and temperature increase. At this point, If $\partial \mathrm{T} / \partial \mathrm{x}>0$, from equation (1) (4), $\mathrm{H}_{\mathrm{h} 1}-\mathrm{H}_{\mathrm{h} 2}>0(\mathrm{H}$ : sensible heat flux; $h_{1}, h_{2}$ : height). Namely, sensible heat flux has increased inside the dry land boundary layer. One side, owing to 
decreased evaporation in the dry land layer, $\partial \mathrm{q} / \partial \mathrm{x}<0$, then $\mathbb{E}_{\mathrm{h} 1}-\mathbb{E}_{\mathrm{h} 2}<0$ (lE: latent heat flux), that is to say, latent heat flux has reduced near the surface. The characteristics of the vertical profile model obtained from our simulation is shown in Fig.4. In area(1)), the flux is constant in the vertical direction (constant flux layer), while in area(2), a new boundary layer has formed, in which the latent heat flux increases with height, and the sensible heat flex decreases with height.

Fluxes are equall

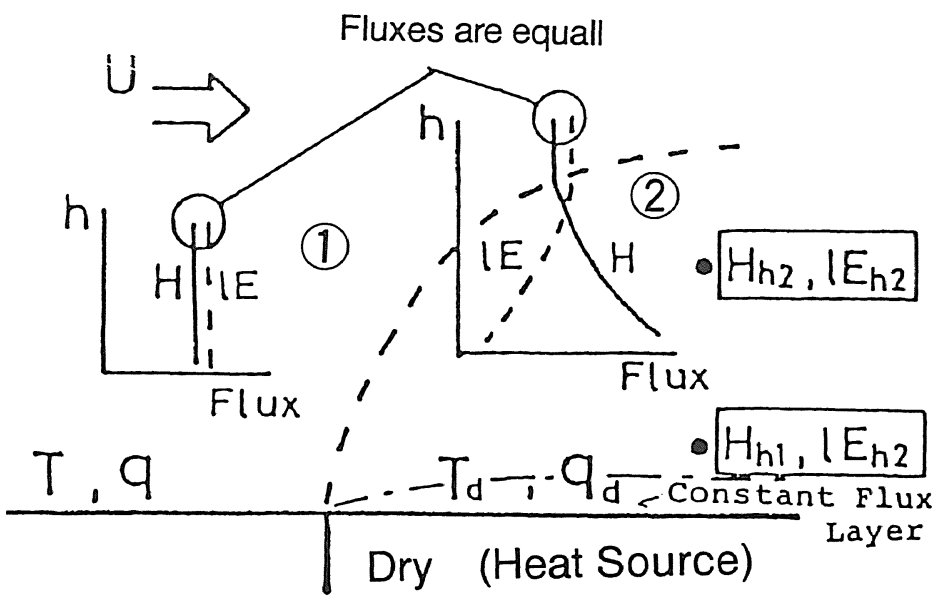

Fig.4 Schematic diagram of the internal boundary layer over different regions. The vertical profiles of sensible latent heat flux are obtained from simulation experiment.

Hayakawa et al.(1981) showed that the observed vertical temperature distribution is very much like the theoretical profiles obtained from the evaluation using $\mathrm{Ri}_{\mathrm{g}}$ in consideration of the stability of each layer, and that the evaluation using a logarithmic profile does not provide a very good fit. These large differences are probably caused by the different ways of determining the diffusion coefficient.

\section{Conclusion}

This paper describes the effects of the localized heat sources(LHS) on the flow and temperature environments when the wind passes from one surface to another with different temperature and humidity.

1) Profiles of the diffusion coefficient $(K)$ obtained by use of $\mathrm{Ri}_{\mathrm{g}}$ show its peaks at reasonable levels compared with these obtained by use of $\mathrm{Ri}_{b}$, and the values of $\mathrm{K}$ and the peak height increase with the scale of LHS.

2) When air moves from a cool area to a hot one, the horizontal temperature gradient $\partial \mathrm{T} / \partial \mathrm{x}$ becomes positive above the hot area, and the gradient of vertical sensible heat flux $\partial \mathrm{H} / \partial \mathrm{z}$ becomes negative above that area. On the other hand, when air moves from a hot area to a cool one, $\partial \mathrm{T} / \partial \mathrm{x}$ becomes negative above the cool area, while $\partial \mathrm{H} / \partial \mathrm{z}$ becomes positive.

\section{References}

Estoque, M.A. and Bhumralkar, C.M., 1970: A method for solving the planetary boundary-layer equations. Boundary-Layer Meteorol., 1, 169-196.

Hayakawa, S., Motoda Y. and Sakanoue T., 1981: A Numerical Study of Mass and Energy Transfer of Surface, Surface Boundary Layer and Planetary Boundary Layer. (II) Advection and its Influence on the Characteristics of Internal Boundary Layers, J. Agric. Meteorol. Japan, 36,237-249.(in Japanese)

Hayakawa, S., Motoda Y. and Sakanoue T., 1985: A Numerical Study of Mass and Energy Transfer of Surface, Surface Boundary Layer and Planetary Boundary Layer. (III) The Characteristics of Internal Boundary Layers Due to the Localized Cool Source Effect, J. Agric. Meteorol. Japan, 40,47-54.(in Japanese)

Blackadar, A., K., 1962: The vertucal distibutioin of wind and turbulent exchange in a nuetral atmosphere. J. Geophys. Res., 67, 3095-3102. 